Review

\title{
Statins in conditions other than hypocholesterolemic effects for chronic subdural hematoma therapy, old drug, new tricks?
}

\author{
Hai Zou ${ }^{1, *}$, Xing-Xing Zhu ${ }^{2, *}$, Ya-Hui Ding ${ }^{1, *}$, Guo-Bing Zhang ${ }^{3}$, Yu Geng ${ }^{4}$ and Dong- \\ Sheng Huang ${ }^{5}$ \\ ${ }^{1}$ Department of Cardiology, Zhejiang Provincial People's Hospital, Hangzhou, China \\ 2 Department of Nephrology, Zhejiang Provincial People's Hospital, Hangzhou, China \\ ${ }^{3}$ Department of Pharmacy, Zhejiang Provincial People's Hospital, Hangzhou, China \\ ${ }^{4}$ Department of Neurology, Zhejiang Provincial People's Hospital, Hangzhou, China \\ ${ }^{5}$ Department of Hepatobiliary Surgery, Zhejiang Provincial People's Hospital, Hangzhou, China \\ * Co-first authors: Hai Zou, Xing-Xing Zhu and Ya-Hui Ding \\ Correspondence to: Guo-Bing Zhang, email: zhangguobing@zjheart.com
}

Yu Geng, email: gengyu@zjheart.com

Dong-Sheng Huang, email: dshuang@zjheart.com

Keywords: statins; chronic subdural hematoma; endothelial progenitor cells; vascular endothelial growth factor; inflammation Received: December 10,2016 Accepted: January 24, $2017 \quad$ Published: January 04, 2017

Copyright: Zou et al. This is an open-access article distributed under the terms of the Creative Commons Attribution License (CC-BY), which permits unrestricted use, distribution, and reproduction in any medium, provided the original author and source are credited.

\section{ABSTRACT}

Chronic subdural hematoma (CSDH) is one of the most common intracranial hematomas worldwide with a high incidence in the general population. However, the optimum treatment for CSDH is Burr-hole drainage with or without rinse Considering the poor outcomes of CSDH in aged patients, and ambiguous prediction of recurrence in many sides of recurrent CSDHs who have been analyzed, new effective therapies are needed for those CSDHs who are predicated to have poor prognosis for surgery and/or have a higher risk of recurrence. Statins, which is the first-line treatment for patients with high cholesterol and coronary heart disease. However, statins are still not solely limited in the treatment of these diseases. It has been demonstrated that statins could improve CSDH due to its effect of regulation of angiogenesis and inflammation. In this review, in order to provide potential new treatment for CSDH we summarize the recent findings of statins in CSDH in order to try to clarify the mechanisms of this effect.

\section{INTRODUCTION}

Chronic subdural hematoma (CSDH) is one of the most common intracranial hematomas worldwide with an incidence of 58 per 10,000 among people 70 years or older and 5 per 10,000 people in the general population [1]. Standard treatment for CSDH is Burr-hole drainage with or without rinsing [2]. However, despite surgical treatment, recurrence rates among treated patients are still relatively high. What's more, as most CSDH patients are elderly, they are at high risk of suffering from pneumonia, peri-operational infection and high-surface-tension pulmonary edema following surgery [3].Considering the poor outcomes of aged patients with CSDH and the likelihood of recurrence, new effective therapies are needed for patients with CSDHs that have a poor prognosis for surgery and/or at high risk for recurrence.
Despite the controversy and ineffectiveness of current therapies and the need for new therapeutic strategies, little is known about the mechanisms underlying CSDH progression [4]. Increasing evidence indicate that impaired angiogenesis in the neomembrane and localized inflammation may play an important role in CSDH formation. Impaired angiogenesis may induce blood leakage from immature vessels of the neomembrane. Repair of vessel leakage is further impeded by localized inflammation [5-8].

Recent studies support a potential therapeutic role for Statins, 3-hydroxy-3-methylglutaryl coenzyme A (HMG-CoA) reductase inhibitors approved for cholesterol reduction, in enhancing angiogenesis and reducing inflammation associated with CSDH $[9,10]$. The statin atorvastatin was shown to improve angiogenesis and increases the level of circulating endothelial progenitor 
cells (EPCs) — cells essential to new blood vessel formation - by activating the endothelial nitric oxide synthase (eNOS), endothelial protein kinase Akt/PKB and Notch1 in patients with CSDH [11-14]. Atorvastatin also inhibited inflammation and decreased the levels of proinflammatory molecules [15-17]. A better understanding of the mechanisms behind these therapeutic statin effects may provide novel targets for future therapeutics for CSDH. In this review, we summarize recent findings regarding the mechanism and role of statins in $\mathrm{CSDH}$.

\section{Statins mediate endothelium renewal and repair via endothelial progenitor cells}

Endothelial progenitor cells (EPCs) are bone marrow-derived late stage stem cells that circulate in the blood. EPCs promote vascular repair of denuded vessel walls and renew endothelium [18] through various processes including mobilization and differentiation [19]. Many factors such as trauma and hematomas mobilize EPCs from the bone into the peripheral bloodstream [20]. These mobile EPCs home to damaged tissue and differentiate into mature endothelial cells that promote vascular repair [21-23].

Song et al. found that the level of peripheral blood EPCs in patients with CSDH was significantly lower than in healthy patients, and that the postoperation EPC level for recurrent patients was significantly lower than in nonrecurrent patients. These data suggest that low EPC levels impair endothelium repair capacity, and thus increase the risk of CSDH development and recurrence [19].

A series of studies have demonstrated that statins promote EPC processes involved in endothelium renewal and repair and reduce senescence and apoptosis. In Landmesser's 2004 study, they showed that increased endothelial nitric oxide (eNO) availability was required for statin-induced improvement of EPC mobilization [24]. Liu et al. showed that atorvastatin blocked miR-221 and miR-222 inhibition of cell migration, tube formation and wound healing by endothelial cells in vitro [25]. In addition, in coronary artery disease patients, miR-221 and miR-222 expression was inversely related to EPC levels [26]. There is also evidence that statins induce mobilization and migration of EPCs through activation of matrix metalloproteinase-2 (MMP-2) and -9 (MMP9), two proteins involved in extracellular matrix (ECM) degradation [27].

Statin promotes EPC activity through multiple pathways. In an ischemic hind limb C57BL/6 mouse model, statins enhanced EPC proliferation and migration and decreased apoptosis through activation of the Akt/ eNOS pathway, effects that were inhibited by the PI3K/ Akt inhibitor, LY294002 [27]. Statin treatment also decreased EPC apoptosis by activation of the Akt/NOS pathway [27]. In a study conducted by Vasa et al, statin treatment activated the Akt/ eNOS pathway and mediated vascular endothelial growth factor (VEGF)-induced EC migration [28]. Statins have also been shown to enhance migration and attenuate cellular senescence by upregulating telomere repeat-binding factor (TRF2) [29]. Statin simvastatin may also promote EPC adhesion by upregulating integrin $\alpha 5$ and $\beta 1$, subunits of the fibronectin receptor [18]. What's more, a recent study showed that, in cultured human ECs, low-dose atorvastatin inhibited EC senescence by suppressing reactive oxygen species (ROS) production [30].

Interestingly, statin-mediated effects are dose dependent. Low doses of statins promoted angiogenesis[10, 28], while high doses of statins inhibited the growth and migration of EPCs [31, 32].

The endothelial promoting effects of statins have revealed the cholesterol lowering therapy to be a potentially novel therapeutic approach to treating $\mathrm{CSDH}$. More researches are needed to further illustrate the functional mechanisms of statins in $\mathrm{CSDH}$ and determine the potential clinical uses for statins for CSDH treatment.

\section{Statins improve vessel maturation by inhibiting vascular endothelial growth factor expression}

Vascular endothelial growth factors (VEGFs) and their receptors play critical roles in vasculogenesis, vascular permeability and angiogenesis [33, 34]. VEGF is also highly expressed in the serum of patients with CSDH and in both the dura and outer membrane of the $\mathrm{CSH}$ $[6,35]$. This high VEGF concentration in the hematoma fluid may be of major pathophysiological importance in the generation and steady increase of the hematoma volume [36]. Interestingly, VEGF promotes angiogenesis, but when expressed persistently at a high level, it also inhibits the maturation of new vessels [37]. This is observed in subdural hematomas. Excessive expression of VEGF increases the number of blood vessels with high permeability [38], but also leads to immature and unstable vessels [39].

A meta-analysis of randomized controlled trials showed that statin treatment was associated with a significant reduction in circulating VEGF concentrations, particularly lipophilic statins and statins administered for over long periods of time [40]. Thus inhibiting VEGF may be beneficial for treating patients with CSDHs in the early stages.

\section{Statins improve functional recovery and vessel maturation by inhibiting inflammation in CSDH}

There is increasing evidence showing that localized inflammation plays a key role in the formation of a CSDH [41-43]. A similar imbalance between anti- and pro-inflammatory molecules has been found in the $\mathrm{CSDH}$ fluid and this imbalance could influence the 
vascular permeability of the hematoma neomembrane and angiogenesis[7, 8, 44, 45]. Recent reports revealed that inflammatory factors, such as TNF- $\alpha$, IL-6 and IL10 are highly expressed in the hematoma fluid of patients with $\mathrm{CSDH}[44,46]$.. The inflammatory reaction alters osmotic pressure of hematoma liquid, and may impact angiogenesis by altering vessel maturation [47].

Several anti-inflammatory therapies are being explored for the treatment of $\mathrm{CSDH}$, including glucocorticoids, mannitol and angiotensin-converting enzyme inhibitors [3] [48-50]. However, these drugs are associated with adverse effects. For example, while dexamethasone, a potent anti-inflammatory drug, successfully cures CSDH [50], it is associated with many side effects such as also cause increased weight, thrush, nausea, agitation, acne, which seriously influenced its clinical use [51].

Statins have also been shown to suppress inflammation and decrease pro-inflammatory molecule levels in CSDH. Li et al. has found that rats with subdermal hematomas (SDHs) treated by atorvastatin experienced better behavioral function recovery and rapid volume elimination, had more new CD31+ blood vessels and less neutrophilic granulocytes than rats treated with saline[52]. Atorvastatin treatment also significantly decreased TNF- $\alpha$ and IL-6 levels [52]. These studies suggest that statininduced inflammation modulation significantly influences SDH elimination and functional recovery in rats [52], and may be beneficial in treating $\mathrm{CSDH}$. More clinical research is needed to confirm the role and the mechanisms that inflammation plays in $\mathrm{CSDH}$.

\section{CONCLUSIONS}

Statins have pleiotropic effects including antiinflammation, neuroprotection and angiogenesis regulation
$[12,15,53]$, and may provide a novel treatment method for $\mathrm{CSDH}$. In this review, we show that statins may impact CSDH development and progression by inhibiting inflammation and improving blood vessel formation and maturation. Evidence from a series of studies showed that statins exhibit ameliorative effects by promoting EPC mobilization and activation, suppressing VEGF and inhibiting inflammation (Figure 1).

ECPs are a key factor in hemostasis and vascular repair through mobilization, tendency, adhesion, proliferation, and differentiation [19]. Statins promote EPC mobilization by increasing eNOS and activation of MMP-2 and -9 [24], enhance EPCs migration by inhibiting miR- 221 and miR-222 [25, 26], enhance EPC proliferation by activating the Akt/eNOS pathway[27, 28] and enhance migratory capacity and attenuate cellular senescence by up-regulating TRF2 [29]. We speculate that statins may improve CSDH via these mechanisms, but more research is still needed to validate these mechanisms and investigate these therapeutic effects.

VEGF is another major component in $\mathrm{CSDH}$ maintenance. VEGF expression is markedly up-regulated in both the dura and outer membrane of CSDH $[35,36]$. Treatment with statins reduces VEGF expression. Thus, treatment with statins in the early stages of a CSDH may prevent VEGF upregulation and further progression of the hematoma [52].

Localized inflammation reaction and inflammatory factors, such as tumor necrosis factor- $\alpha$ (TNF- $\alpha$ ), interleukin-6 (IL-6) and IL-10, also contribute to CSDH formation [41-43, 46]. Statin-induced inflammatory modulation significantly improved functional recover, SDH elimination, and vessel maturation in rats with CSDH Treatment also significantly decreased TNF- $\alpha$ and IL-6 expression [52].

In conclusion, statins are a promising therapeutic intervention for the treatment of CSDH. More research is

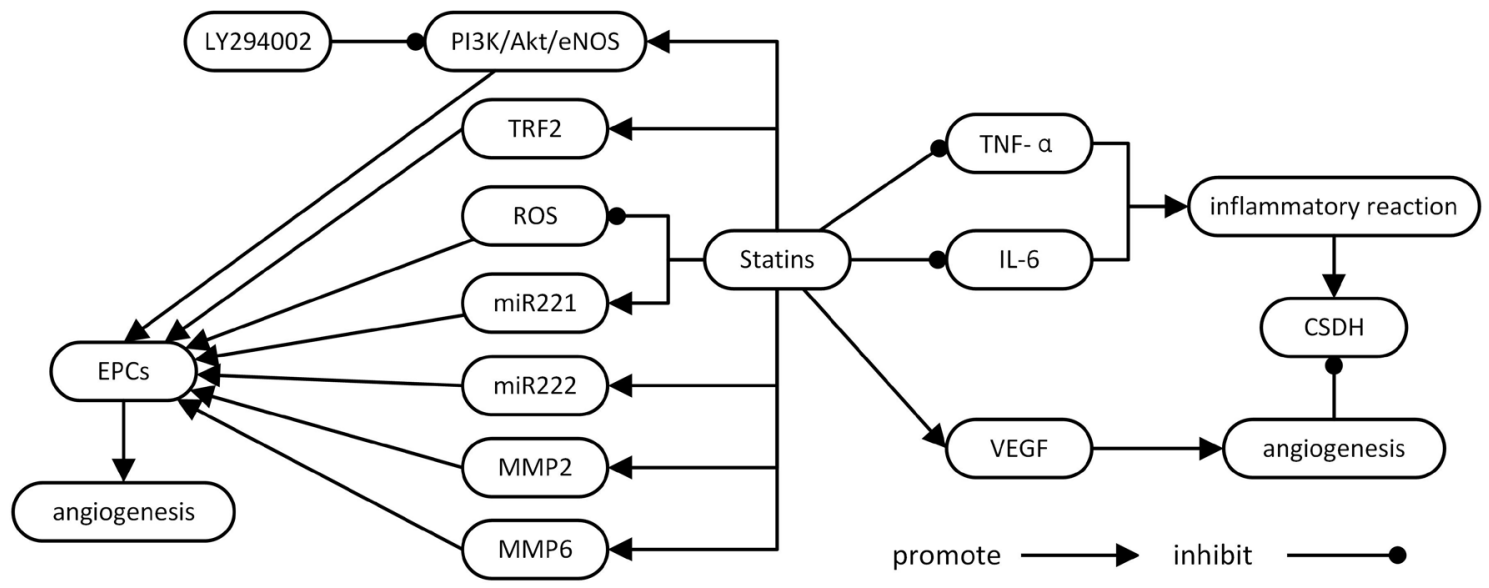

Figure 1: Potential mechanisms and the role of statins in CSDH. CSDH: chronic subdural hematoma; EPCs: endothelial progenitor cells; IL-6: interleukin-6; PI3K/Akt/eNOS: phosphatidylinositol 3-kinase/ protein kinase B/ endothelial nitric oxide synthase; ROS: reactive oxygen species; TRF2: TTAGGG repeat binding factor-2; TNF- $\alpha$ : tumor necrosis factor $-\alpha$; VEGF: vascular endothelial growth factor. 
needed to further illustrate the functional mechanisms of statins in $\mathrm{CSDH}$.

\section{EXPERT OPINION}

In this report, we have discussed the therapeutic potential of statins in treating CSDH based on studies conducted in the last five years. We have discovered several interesting findings on how statins may influence CSDH. The first one being that different doses of stains have different effects on EPCs and VEGF. Low doses of statins promote angiogenesis [10, 28], but high doses inhibit EPC growth and migration which inhibits angiogenesis [31, 32]. Interestingly, VEGF promotes angiogenesis, but when expressed at a high level it also inhibits the maturation of new vessels [37]. These dosesdependent effects need further investigation.

The second interesting finding is that stains may have varying effects via VEGF in different stages of CSDH. Li et al. found that VEGF expression was high and then slowly decreased following treatment with a statin [52]. We speculate that, in early CSDH, inhibiting VEGF may be beneficial for preventing further $\mathrm{CSDH}$ development.

While an increasing number of studies are exploring the role of statins in $\mathrm{CSDH}$, it is important that the biological and underlying mechanisms of its effects remain a major focus of future studies.

\section{FIVE-YEAR VIEW}

A randomized clinical trial about the effect and safety of atorvastatin to treat CSDH in ClinicTrials.gov (NCT0236232) has been completed in October, 2016. Excitedly, the result of atorvastatin on CSDH was positive in this study. We are waiting for the publication of this important article. Over the next 5 years, we expect statins to be a strong contender as a therapeutic for CSDH. Pharmacological evidence and clinical trial results support the interpretation that statins treat CSDHs by regulating potential signaling pathways, such as Akt/eNOS pathway, MMP-2 and MMP-9,ROS, TNF- $\alpha$ and IL-6.We predict that statins will be approved for the treatment of these CSDHs. Further research is need to confirm the mechanism, therapeutic effects and safety of statin use in treating $\mathrm{CSDH}$.

\section{Key points}

- Different doses of stains have different effects on EPCs and VEGF. Low dose of statins promoted angiogenesis, but high dose of statins inhibited the growth and migration of EPCs to inhibit angiogenesis.

- Stains may has different role via VEGF in different stage of CSDH. In early CSDH, inhibiting VEGF may be benefit for the occurrence of $\mathrm{CSDH}$, but in late $\mathrm{CSDH}$, increasing VEGF may be benefit for the occurrence of CSDH.

\section{ACKNOWLEDGMENTS}

There was no financial support or funding for this article.

\section{CONFLICTS OF INTEREST}

All authors: no conflicts.

\section{Authors' contributions}

Hai Zou and Guo-Bing Zhang planned the article and contributed to data collection, discussing content, writing and reviewing the article. Xing-Xing Zhu, Ya-Hui Ding, Dong-Sheng Huang and Yu Geng participated in its design, study supervision and helping to writing the article.

\section{REFERENCES}

1. Emich S, Richling B, McCoy MR, Al-Schameri RA, Ling F, Sun L, Wang Y, Hitzl W. The efficacy of dexamethasone on reduction in the reoperation rate of chronic subdural hematoma - the DRESH study: straightforward study protocol for a randomized controlled trial. Trials. 2014; $15: 6$.

2. Muzii VF, Bistazzoni S, Zalaffi A, Carangelo B, Mariottini A, Palma L. Chronic subdural hematoma: comparison of two surgical techniques. Preliminary results of a prospective randomized study. J Neurosurg Sci. 2005; 49:41-46.

3. Santarius T, Hutchinson PJ. Chronic subdural haematoma: time to rationalize treatment? $\mathrm{Br} \mathrm{J}$ Neurosurg. 2004; 18:328-32.

4. Weigel R, Schmiedek P, Krauss JK. Outcome of contemporary surgery for chronic subdural haematoma: evidence based review. J Neurol Neurosurg Psychiatry. 2003; 74:937-43.

5. Javadi A, Amirjamshidi A, Aran S, Hosseini SH. A randomized controlled trial comparing the outcome of burrhole irrigation with and without drainage in the treatment of chronic subdural hematoma: a preliminary report. World Neurosurg. 2011; 75:731-36.

6. Hohenstein A, Erber R, Schilling L, Weigel R. Increased mRNA expression of VEGF within the hematoma and imbalance of angiopoietin-1 and -2 mRNA within the neomembranes of chronic subdural hematoma. J Neurotrauma. 2005; 22:518-28.

7. Stanisic M, Lyngstadaas SP, Pripp AH, Aasen AO, Lindegaard KF, Ivanovic J, Ilstad E, Konglund A, Sandell T, Ellingsen 
O, Saehle T. Chemokines as markers of local inflammation and angiogenesis in patients with chronic subdural hematoma: a prospective study. Acta Neurochir (Wien). 2012; 154:113-20.

8. Stanisic M, Aasen AO, Pripp AH, Lindegaard KF, RammPettersen J, Lyngstadaas SP, Ivanovic J, Konglund A, Ilstad E, Sandell T, Ellingsen O, Sæhle T. Local and systemic pro-inflammatory and anti-inflammatory cytokine patterns in patients with chronic subdural hematoma: a prospective study. Inflamm Res. 2012; 61:845-52.

9. Youssef S, Stüve O, Patarroyo JC, Ruiz PJ, Radosevich JL, Hur EM, Bravo M, Mitchell DJ, Sobel RA, Steinman L, Zamvil SS. The HMG-CoA reductase inhibitor, atorvastatin, promotes a Th2 bias and reverses paralysis in central nervous system autoimmune disease. Nature. 2002; 420:78-84.

10. Kureishi Y, Luo Z, Shiojima I, Bialik A, Fulton D, Lefer DJ, Sessa WC, Walsh K. The HMG-CoA reductase inhibitor simvastatin activates the protein kinase Akt and promotes angiogenesis in normocholesterolemic animals. Nat Med. 2000; 6:1004-10.

11. Kikuchi R, Takeshita K, Uchida Y, Kondo M, Cheng XW, Nakayama T, Yamamoto K, Matsushita T, Liao JK, Murohara T. Pitavastatin-induced angiogenesis and arteriogenesis is mediated by Notch1 in a murine hindlimb ischemia model without induction of VEGF. Lab Invest. 2011; 91:691-703.

12. Wang B, Sun L, Tian Y, Li Z, Wei H, Wang D, Yang Z, Chen J, Zhang J, Jiang R. Effects of atorvastatin in the regulation of circulating EPCs and angiogenesis in traumatic brain injury in rats. J Neurol Sci. 2012; 319:117-23.

13. Lu D, Qu C, Goussev A, Jiang H, Lu C, Schallert T, Mahmood A, Chen J, Li Y, Chopp M. Statins increase neurogenesis in the dentate gyrus, reduce delayed neuronal death in the hippocampal CA3 region, and improve spatial learning in rat after traumatic brain injury. J Neurotrauma. 2007; 24:1132-46.

14. Matsumura M, Fukuda N, Kobayashi N, Umezawa H, Takasaka A, Matsumoto T, Yao EH, Ueno T, Negishi N. Effects of atorvastatin on angiogenesis in hindlimb ischemia and endothelial progenitor cell formation in rats. $\mathrm{J}$ Atheroscler Thromb. 2009; 16:319-26.

15. Araújo FA, Rocha MA, Mendes JB, Andrade SP. Atorvastatin inhibits inflammatory angiogenesis in mice through down regulation of VEGF, TNF-alpha and TGFbeta1. Biomed Pharmacother. 2010; 64:29-34.

16. Buttmann M, Lorenz A, Weishaupt A, Rieckmann P. Atorvastatin partially prevents an inflammatory barrier breakdown of cultured human brain endothelial cells at a pharmacologically relevant concentration. J Neurochem. 2007; 102:1001-08.

17. Jaiswal SR, Sontakke SD. Experimental evaluation of analgesic and anti-inflammatory activity of simvastatin and atorvastatin. Indian J Pharmacol. 2012; 44:475-79.
18. Walter DH, Rittig K, Bahlmann FH, Kirchmair R, Silver M, Murayama T, Nishimura H, Losordo DW, Asahara T, Isner JM. Statin therapy accelerates reendothelialization: a novel effect involving mobilization and incorporation of bone marrow-derived endothelial progenitor cells. Circulation. 2002; 105:3017-24.

19. Song Y, Wang Z, Liu L, Wang D, Zhang J. The level of circulating endothelial progenitor cells may be associated with the occurrence and recurrence of chronic subdural hematoma. Clinics (Sao Paulo). 2013; 68:1084-88.

20. Liu S, Liu T, Chen J, Maitz M, Chen C, Huang N. Influence of a layer-by-layer-assembled multilayer of anti-CD34 antibody, vascular endothelial growth factor, and heparin on the endothelialization and anticoagulation of titanium surface. J Biomed Mater Res A. 2013; 101:1144-57.

21. Li W, Wang H, Kuang CY, Zhu JK, Yu Y, Qin ZX, Liu J, Huang L. An essential role for the Id1/PI3K/Akt/NFkB/ survivin signalling pathway in promoting the proliferation of endothelial progenitor cells in vitro. Mol Cell Biochem. 2012; 363:135-45.

22. Caporali A, Pani E, Horrevoets AJ, Kraenkel N, Oikawa A, Sala-Newby GB, Meloni M, Cristofaro B, Graiani G, Leroyer AS, Boulanger CM, Spinetti G, Yoon SO, et al. Neurotrophin $\mathrm{p} 75$ receptor ( $\mathrm{p} 75 \mathrm{NTR}$ ) promotes endothelial cell apoptosis and inhibits angiogenesis: implications for diabetes-induced impaired neovascularization in ischemic limb muscles. Circ Res. 2008; 103:e15-26.

23. Miller-Kasprzak E, Jagodziński PP. Endothelial progenitor cells as a new agent contributing to vascular repair. Arch Immunol Ther Exp (Warsz). 2007; 55:247-59.

24. Landmesser U, Engberding N, Bahlmann FH, Schaefer A, Wiencke A, Heineke A, Spiekermann S, Hilfiker-Kleiner D, Templin C, Kotlarz D, Mueller M, Fuchs M, Hornig B, et al. Statin-induced improvement of endothelial progenitor cell mobilization, myocardial neovascularization, left ventricular function, and survival after experimental myocardial infarction requires endothelial nitric oxide synthase. Circulation. 2004; 110:1933-39.

25. Liu Y, Wei J, Hu S, Hu L. Beneficial effects of statins on endothelial progenitor cells. Am J Med Sci. 2012; 344:220 26.

26. Minami Y, Satoh M, Maesawa C, Takahashi Y, Tabuchi T, Itoh T, Nakamura M. Effect of atorvastatin on microRNA $221 / 222$ expression in endothelial progenitor cells obtained from patients with coronary artery disease. Eur J Clin Invest. 2009; 39:359-67.

27. Shao H, Tan Y, Eton D, Yang Z, Uberti MG, Li S, Schulick A, Yu H. Statin and stromal cell-derived factor-1 additively promote angiogenesis by enhancement of progenitor cells incorporation into new vessels. Stem Cells. 2008; 26:137684.

28. Llevadot J, Murasawa S, Kureishi Y, Uchida S, Masuda H, Kawamoto A, Walsh K, Isner JM, Asahara T. HMGCoA reductase inhibitor mobilizes bone marrow-derived endothelial progenitor cells. J Clin Invest. 2001; 108:399- 
405.

29. Spyridopoulos I, Haendeler J, Urbich C, Brummendorf TH, Oh H, Schneider MD, Zeiher AM, Dimmeler S. Statins enhance migratory capacity by upregulation of the telomere repeat-binding factor TRF2 in endothelial progenitor cells. Circulation. 2004; 110:3136-42.

30. Satoh M, Minami Y, Takahashi Y, Tabuchi T, Itoh T, Nakamura M. Effect of intensive lipid-lowering therapy on telomere erosion in endothelial progenitor cells obtained from patients with coronary artery disease. Clin Sci (Lond). 2009; 116:827-35.

31. Feng C, Han A, Ye C, Xu R, Li M. The HMG-CoA reductase pathway, statins and angioprevention. Semin Ophthalmol. 2006; 21:29-35.

32. Weis M, Heeschen C, Glassford AJ, Cooke JP. Statins have biphasic effects on angiogenesis. Circulation. 2002; 105:739-45.

33. Ferrara N, Gerber HP, LeCouter J. The biology of VEGF and its receptors. Nat Med. 2003; 9:669-76.

34. Takahashi H, Shibuya M. The vascular endothelial growth factor (VEGF)/VEGF receptor system and its role under physiological and pathological conditions. Clin Sci (Lond). 2005; 109:227-41.

35. Saito A, Narisawa A, Takasawa H, Morita T, Sannohe S, Sasaki T, Kurotaki H, Nishijima M. Expression of the TGF- $\beta$-ALK- 1 pathway in dura and the outer membrane of chronic subdural hematomas. Neurol Med Chir (Tokyo). 2014; 54:357-62.

36. Hua C, Zhao G, Feng Y, Yuan H, Song H, Bie L. Role of Matrix Metalloproteinase-2, Matrix Metalloproteinase-9, and Vascular Endothelial Growth Factor in the Development of Chronic Subdural Hematoma. J Neurotrauma. 2016; 33:65-70.

37. Nagy JA, Vasile E, Feng D, Sundberg C, Brown LF, Manseau EJ, Dvorak AM, Dvorak HF. VEGF-A induces angiogenesis, arteriogenesis, lymphangiogenesis, and vascular malformations. Cold Spring Harb Symp Quant Biol. 2002; 67:227-37.

38. Tsigkos S, Koutsilieris M, Papapetropoulos A. Angiopoietins in angiogenesis and beyond. Expert Opin Investig Drugs. 2003 ; $12: 933-41$.

39. Yancopoulos GD, Davis S, Gale NW, Rudge JS, Wiegand SJ, Holash J. Vascular-specific growth factors and blood vessel formation. Nature. 2000; 407:242-48.

40. Sahebkar A, Ponziani MC, Goitre I, Bo S. Does statin therapy reduce plasma VEGF levels in humans? A systematic review and meta-analysis of randomized controlled trials. Metabolism. 2015; 64:1466-76.

41. Schachenmayr W, Friede RL. The origin of subdural neomembranes. I. Fine structure of the dura-arachnoid interface in man. Am J Pathol. 1978; 92:53-68.
42. Yamashima T, Yamamoto S. The origin of inner membranes in chronic subdural hematomas. Acta Neuropathol. 1985; 67:219-25.

43. Moskala M, Goscinski I, Kaluza J, Polak J, Krupa M, Adamek D, Pitynski K, Miodonski AJ. Morphological aspects of the traumatic chronic subdural hematoma capsule: SEM studies. Microsc Microanal. 2007; 13:21119.

44. Wada T, Kuroda K, Yoshida Y, Ogasawara K, Ogawa A, Endo S. Local elevation of the anti-inflammatory interleukin-10 in the pathogenesis of chronic subdural hematoma. Neurosurg Rev. 2006; 29:242-45.

45. Wang D, Li T, Wei H, Wang Y, Yang G, Tian Y, Zhao Z, Wang L, Yu S, Zhang Y, Chen J, Jiang R, Zhang JN. Atorvastatin enhances angiogenesis to reduce subdural hematoma in a rat model. J Neurol Sci. 2016; 362:91-99.

46. Suzuki M, Endo S, Inada K, Kudo A, Kitakami A, Kuroda $\mathrm{K}$, Ogawa A. Inflammatory cytokines locally elevated in chronic subdural haematoma. Acta Neurochir (Wien). 1998; 140:51-55

47. Glover LE, Colgan SP. Hypoxia and metabolic factors that influence inflammatory bowel disease pathogenesis. Gastroenterology. 2011; 140:1748-55.

48. Parlato C, Guarracino A, Moraci A. Spontaneous resolution of chronic subdural hematoma. Surg Neurol. 2000; 53:31215.

49. Weigel R, Hohenstein A, Schlickum L, Weiss C, Schilling L. Angiotensin converting enzyme inhibition for arterial hypertension reduces the risk of recurrence in patients with chronic subdural hematoma possibly by an antiangiogenic mechanism. Neurosurgery. 2007; 61:788-92.

50. Delgado-López PD, Martín-Velasco V, Castilla-Díez JM, Rodríguez-Salazar A, Galacho-Harriero AM, FernándezArconada O. Dexamethasone treatment in chronic subdural haematoma. Neurocirugia (Astur). 2009; 20:346-59.

51. Vardy J, Chiew KS, Galica J, Pond GR, Tannock IF. Side effects associated with the use of dexamethasone for prophylaxis of delayed emesis after moderately emetogenic chemotherapy. Br J Cancer. 2006; 94:1011-15.

52. Li T, Wang D, Tian Y, Yu H, Wang Y, Quan W, Cui W, Zhou L, Chen J, Jiang R, Zhang J. Effects of atorvastatin on the inflammation regulation and elimination of subdural hematoma in rats. J Neurol Sci. 2014; 341:88-96.

53. Wang Y, Chang H, Zou J, Jin X, Qi Z. The effect of atorvastatin on mRNA levels of inflammatory genes expression in human peripheral blood lymphocytes by DNA microarray. Biomed Pharmacother. 2011; 65:118-22. 